\title{
Equipos biomédicos coadyuvantes para el control de la nutrición y para el ejercicio físico de personas con diabetes
}

\section{(Biomedical equipment for the control of nutrition and for the physical exercise of people with diabetes)}

\author{
Mantilla Guerra Aníbal Rubén¹
}

\begin{abstract}
Resumen:
Con el fin de coadyuvar en el tratamiento de la diabetes, que es una de las principales causas de muerte en el Ecuador y en el mundo, se está desarrollando un Proyecto de Investigación para crear equipos que permitan el control y tratamiento efectivo de esta enfermedad. El equipo electrónico creado para el control de la nutrición, permite que el enfermo diabético cuente con la información sobre la cantidad de calorías que debe ingerir en consideración de sus actividades y su tratamiento médico. El equipo mecatrónico para el ejercicio físico de personas con pie diabético, permite aplicar terapias en función de la severidad de la afectación, considerando lo delicado y complejo que puede ser dicho tratamiento, para así, aliviar su malestar, disminuir el tiempo de recuperación, y en algunos casos incluso evitar la amputación. El equipo biomédico para el control de la nutrición ha sido completamente terminado; mientras que el equipo para el tratamiento del pie diabético se encuentra en etapas de desarrollo de prototipos y pruebas. Los dos equipos han sido concebidos y desarrollados con rigurosos métodos de ingeniería bajo estándares de calidad, por lo cual constituyen un aporte importante a la investigación, desarrollo de ciencia, tecnología e innovación.
\end{abstract}

Palabras clave: Diabetes; equipo biomédico; nutrición; pie diabético; ejercitador

\begin{abstract}
:
In order to contribute to the treatment of diabetes, which is one of the main causes of death in Ecuador and in the world, a research project is being developed to create equipment that allows the effective control and treatment of this disease. The electronic equipment created for nutrition control, allows the diabetic patient to have information about the amount of calories they should ingest in consideration of their activities and their medical treatment. The mechatronic equipment for the physical exercise of people with diabetic foot, allows the application of therapies according to the severity of the affection. Considering that this is a delicate and complex treatment, the equipment helps the patients to alleviate its discomfort, to decrease the time of recovery and in some cases even to avoid amputation. The construction of the biomedical equipment for the control of nutrition is completed. While the equipment for the treatment of diabetic foot is in a stage of development of prototypes and tests. Both equipment has been conceived and developed with rigorous engineering methods and under quality standards, making an important contribution to research, development of science, technology, and innovation.
\end{abstract}

Keywords: Diabetes; biomedical equipment; nutrition; diabetic foot; exerciser

\footnotetext{
1 Universidad Tecnológica Equinoccial, Quito - Ecuador (anibal.mantilla@ute.edu.ec)
} 


\section{Introducción}

La Diabetes Mellitus es un grupo de alteraciones metabólicas que se caracteriza por hiperglucemia crónica debida a un defecto en la secreción de insulina, a un defecto en la acción de la misma o a ambas. Además de la hiperglucemia coexisten alteraciones en el metabolismo de las grasas y de las proteínas. Cuando se expresa en su plenitud, la diabetes se caracteriza por hiperglucemia en ayunas, pero la enfermedad también se reconoce en las etapas menos manifiestas, principalmente por la presencia de intolerancia a la glucosa. La hiperglucemia sostenida en el tiempo se asocia con daño, disfunción y colapso a largo plazo de varios órganos y sistemas, especialmente los ojos, los riñones, los nervios, el corazón y los vasos sanguíneos; y la ausencia de un tratamiento eficaz, podría provocar inclusive estupor, coma y hasta la muerte (Proctor, 2007, pág. 331).

La diabetes es considerada una enfermedad crónica debido a su larga evolución y progresión lenta. Sus efectos a corto y largo plazo son enormes y repercuten no solo en quien la padece, sino también en la familia, en la sociedad e inclusive en el Sistema de Salud. La prevención es la clave fundamental para evitar esta terrible enfermedad, así como sus complicaciones y consecuencias (WHO \& FAO, 2003).

En la actualidad la Diabetes es una pandemia. Según datos de la OMS, es una de las 10 principales causas de muerte en el mundo. Se estima que en el período comprendido entre los años 2015 y 2040 habrá un incremento de enfermos diabéticos de hasta un 55\% como se indica en la Tabla 1 (FID, 2015, pág. 89).

Tabla 1. Cifras actuales y proyectadas de población mundial diabética en millones de habitantes entre 20 - 79 Años

\begin{tabular}{|c|c|c|}
\hline Población Mundial con diabetes & $\begin{array}{c}\text { Año } \\
\mathbf{2 0 1 5}\end{array}$ & $\begin{array}{c}\text { Año } \\
\mathbf{2 0 4 0}\end{array}$ \\
\hline $20-64$ años & 320.5 & 441.3 \\
\hline $65-79$ años & 94.2 & 200.5 \\
\hline Total en millones & 414.7 & 641.8 \\
\hline
\end{tabular}

En Ecuador, en la población comprendida entre los 20 a 79 años de edad, existen 830.100 personas diagnosticadas de diabetes y 332.300 que podrían no estar diagnosticadas, con lo cual, la cifra total de enfermos diabéticos podría alcanzar a 1,136.000 de personas. De acuerdo a las cifras de mortalidad en el Ecuador durante el año 2014, la diabetes es la primera causa de muerte de mujeres, y la segunda total a nivel nacional (INEC, 2014); como se aprecia en la Tabla 2.

Tabla 2. Principales causas de muerte en la población del Ecuador, clasificado por enfermedad y género, año 2014

\begin{tabular}{|l|c|c|c|}
\hline \multicolumn{1}{|c|}{ Enfermedad } & Hombres & Mujeres & Total \\
\hline Diabetes & 2.030 & 2.371 & 4.401 \\
\hline Corazón & 2.643 & 1.787 & 4.430 \\
\hline Hipertensión & 1.698 & 1.874 & 3.572 \\
\hline Cerebrovascular & 1.886 & 1.891 & 3.777 \\
\hline
\end{tabular}


Si el total de fallecidos en el Ecuador en 365 días es de 4.401, y tomando en cuenta que el año tiene 8.760 horas, estadísticamente se puede decir que cada 2 horas muere una persona a causa de la diabetes.

Los tratamientos pueden ser muy rigurosos y además muy costosos, y posiblemente los Sistemas de Salud Pública y Privada, no están en la capacidad de tratar a todos los enfermos de diabetes, por lo que es fundamental que el enfermo diabético cuide su salud con una buena nutrición (Joslin, 2016, pág. 612) que esté adecuada a su condición médica, tratamiento y actividad física (García Yanes, 2009).

Considerando la Innovación Científica y Tecnológica como un factor indispensable para el desarrollo y bienestar de la Sociedad, se planeó la creación de equipos biomédicos inéditos, que, considerando estándares de calidad, permitan a las personas con diabetes, tener conocimiento de la ingesta calórica que necesita día a día y por tanto una nutrición, que le permita mantener control sobre sus niveles de glucosa.

Por otra parte, una complicación crónica de la diabetes mellitus es el pie diabético, enfermedad que presenta neuropatías sensitivo motoras, angiopatía, edema y afectación inmunológica casi siempre graves, cuyas consecuencias pueden ser infecciones, ulceración y gangrena que implican hospitalización o cirugía que involucra amputaciones que podrían provocar incapacidad parcial o definitiva del paciente. En numerosos estudios clínicos se ha encontrado pruebas de que la neuropatía diabética es la más común y grave de las complicaciones crónicas de pacientes diabéticos no controlados, sin embargo, es posible en cierta medida, prever y evitar dichas complicaciones, con un control estricto de la glucemia, la exploración rutinaria del pie, y la realización constante de ejercicio. Aproximadamente el $20 \%$ de los diabéticos presentará un cuadro de pie diabético, en el transcurso de su vida, de los cuales aproximadamente $20 \%$ de los casos terminará en amputación. (Jesús, 2010, págs. 73-74) . El enfermo que sufra de pie diabético, podrá con la ayuda del equipo, realizar terapias que mejoran su condición con lo cual puede aliviar su malestar, disminuir el tiempo de recuperación, y en algunos casos incluso puede evitar la amputación.

La forma en que está organizado el documento es el siguiente: etapas de desarrollo de los equipos biomédicos, resultados alcanzados, conclusiones.

\subsection{Etapas de desarrollo de los equipos biomédicos}

El equipo electrónico para el control de la nutrición, ha sido desarrollado en las siguientes etapas:

- Materiales y métodos : determinación del requerimiento calórico, diseño y construcción del equipo, definición del prototipo

- Diseño del hardware y software, que comprende: diseño del hardware, diseño del software, construcción del prototipo físico 
Las etapas bajo la cuales ha sido desarrollado el equipo mecatrónico para el ejercicio físico de personas con diabetes, son las siguientes:

- Materiales y métodos: determinación del grado de afectación del pie diabético, tratamiento del pie diabérico, diseño y construcción del equipo, definición del prototipo

- Diseño del hardware, del software y del sistema mecánico, que comprende: diseño del hardware, diseño del software, diseño virtual del sistema mecánico

\subsection{Resultados alcanzados}

Una vez presentadas las etapas de desarrollo, se expone los resultados alcanzados en cada caso con su respectivo análisis. Los resultados se encuentran organizados de la siguiente manera:

- Resultado: equipo biomédico electrónico para el control de la nutrición. El usuario es el paciente diabético.

- Resultado: prototipo físico de equipo biomédico mecatrónico para el ejercicio de personas con diabetes. Su uso permitirá a los investigadores, continuar con el proceso de construcción del equipo que será para uso del paciente.

Posteriormente a la presentación de los resultados alcanzados con su respectivo análisis, se presenta las conclusiones para cada uno de los dos equipos.

\section{Desarrollo de los equipos biomédicos coadyuvantes para el control de la nutrición de personas con diabetes y el tratamiento del pie diabético}

\subsection{Desarrollo del equipo para el control de la nutrición de personas con diabetes}

\subsubsection{Materiales y métodos}

\subsubsection{Determinación del requerimiento calórico}

Es fundamental determinar y valorar el estado nutricional de la persona, establecer el valor nutricional de los alimentos, conocer la cantidad de calorías requeridas y que se consumen en función de la actividad, en concordancia con la medicación establecida por el médico. Todo esto orientado en base a las posibles necesidades de las personas diabéticas, según recomendaciones de médicos especialistas en el tema e información de instituciones y organismos con trascendencia mundial, como la Organización Mundial de la Salud, la International Diabetes Federation (IDF, 2015, pág. 8), y el centro médico más importante del mundo dedicado a la investigación y tratamiento de la diabetes, el Joslin Diabetes Center. Es necesario realizar el cálculo del índice de masa corporal, de la tasa metabólica basal, y el cálculo del gasto calórico. La manera de calcular estas variables, se presenta a continuación. 


$$
\mathrm{IMC}=\frac{\text { Peso }[\mathrm{Kg}]}{\text { Talla }^{2}\left[\mathrm{~m}^{2}\right]}
$$

Dónde; Peso: Peso de la persona en Kilogramos

Talla: Estatura de la persona en metros

La tasa metabólica basal (TMB) determina la cantidad de calorías diarias necesarias para mantener las funciones básicas del cuerpo (Lineaysalud, 2009). Su cálculo se realiza según la ecuación 2.

$$
\mathrm{TMB}=(10 \mathrm{x} \text { peso }[\mathrm{Kg}])+(6,25 \mathrm{x} \text { altura }[\mathrm{cm}])-(5 \mathrm{x} \text { edad }[\operatorname{años}])+\mathrm{X}
$$

Siendo $\mathrm{X}$ igual a 5 para el caso de hombres y menos 161 para mujeres.

La ecuación de Harris Benedict (Villarino, 2016) se utiliza para estimar el metabolismo basal de una persona en función de su peso corporal, estatura y edad, es manejada en conjunto con factores de actividad física para calcular el consumo diario de calorías.

Para el cálculo de la ingesta diaria de calorías recomendada según el principio de Harris Benedict para mantener el peso actual y realizando una determinada actividad física se multiplica la TMB por una constante que varía según la intensidad del ejercicio realizado como se indica en la Tabla 3.

Tabla 3. Ingesta diaria de calorías en función de la intensidad del ejercicio.

\begin{tabular}{|l|l|}
\hline \multicolumn{1}{|c|}{ Ejercicio } & Ingesta diaria de calorías \\
\hline Poco o ningún ejercicio & $\mathrm{TMB} \times 1,2$ \\
\hline Ligero (1-3 días por semana) & $\mathrm{TMB} \times 1,375$ \\
\hline Moderado (3-5 días por semana) & $\mathrm{TMB} \times 1,55$ \\
\hline Fuerte (6-7 días por semana) & $\mathrm{TMB} \times 1,725$ \\
\hline Muy fuerte (dos veces al día) & $\mathrm{TMB} \times 1,9$ \\
\hline
\end{tabular}

Un MET es la cantidad de calor emitido por una persona, en posición sedente por metro cuadrado de piel. A partir de este estado, se incrementan los MET's en medida que aumenta la intensidad de la actividad. El gasto calórico, basado en la intensidad MET's, se calcula con la ecuación 3.

Gasto de calorías $[\mathrm{Kcal}]=($ MET's x 3,5 x Masa corporal $[\mathrm{Kg}] / 200) \times$ tiempo[minutos]

\subsubsection{Diseño y construcción del equipo para el control de la nutrición}

En cuanto a la tecnología de hardware y software en el mundo, ésta ha ido evolucionando de manera vertiginosa, llegando en la actualidad al punto en que es posible desarrollar dispositivos portables de altas prestaciones y de fácil manejo para el usuario. La medicina se ha visto favorecida en este caso por estos avances tecnológicos. Esto se ha aprovechado para el desarrollo del equipo biomédico para diabéticos. 
Las especificaciones técnicas y de operación del equipo son las siguientes:

- Por medio de una balanza y un teclado, el usuario ingresa información que es procesada por el equipo, para recibir como resultado, información sobre las calorías que requiere para su nutrición y consumo. Adicionalmente presentará en forma gráfica, el registro histórico de glucemia y peso. Este equipo debe ser portable.

- El programa calculará el índice de masa corporal, la ingesta y consumo de calorías, en función de la valoración nutricional del paciente, tasa metabólica basal, y el cálculo de los MET's, tomando en cuenta la intensidad y duración de la actividad que realiza. El software deberá ser amigable con el usuario.

- El equipo tendrá un microcontrolador con capacidad para manejar una pantalla táctil a color, almacenar datos, y recibir señales provenientes de una balanza electrónica. Deberá contar además con un reloj en tiempo real.

\subsubsection{Definición del prototipo}

Partiendo del uso de microcontroladores, funciones e interfaces se construye prototipos básicos, que progresivamente escalados permiten elaborar el prototipo final sobre el cual se concibe el equipo para el usuario.

\subsubsection{Diseño del hardware y software}

Aplicando la metodología descrita, se diseñó el hardware y el software que sería embebido en él, para gestionar todos los recursos del equipo. Como resultado, se construyó un prototipo bajo especificaciones y en consideración de los requisitos de las dos normas antes mencionadas.

\subsubsection{Diseño hardware}

La balanza puede medir en un rango de $0.5 \mathrm{~g}$ a $3 \mathrm{Kg}$, la señal que la medición produce es entregada al módulo de pesaje HX711. Considerando los requisitos de las normas, se optó por utilizar una fuente de alimentación externa de DC, de 3,3 V a 5 V, un AVR XMEGA 256 D3 que trabaja a $32 \mathrm{MHz}$, una tarjeta controladora de pantalla gráfica SSD1963. La Figura 1, presenta los componentes del hardware y la forma en que ellos se integran para recibir información de entrada, procesarla, y presentar información al usuario. Las flechas de colores diferentes, muestran como fluyen, la energía eléctrica de alimentación, los datos, la información y las señales de control. 

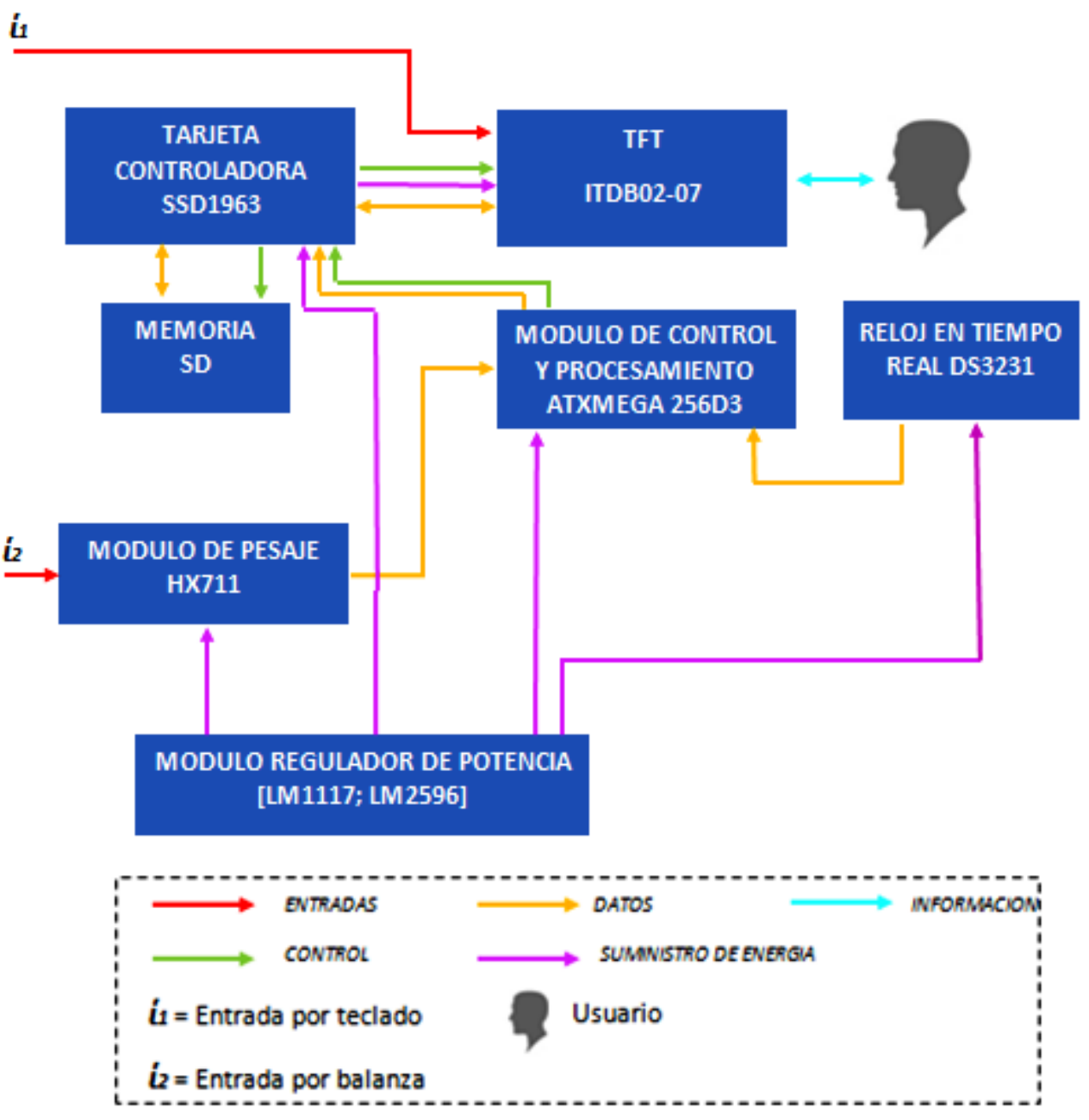

Figura 1. Diagrama de bloques de operación e integración del hardware - equipo para el control de la nutrición

\subsubsection{Diseño del software}

Se utilizó el lenguaje de programación BASCOM, en el IDE ATMEL STUDIO 4.0, porque este lenguaje posee numerosas y potentes herramientas para el manejo de una pantalla de cristal líquido táctil.

MCS electronics, recomienda BASCOM para la programación del microcontrolador XMEGA 256 D3, razón por la cual se escogió este lenguaje.

El software se diseñó considerando que el microcontrolador debe gestionar todos los recursos de hardware, para adquisición, almacenamiento y manejo de datos, realizar cálculos y presentar información. La Figura 2, muestra el diagrama de flujo diseñado para el software del equipo. Para el diseño de varias interfaces graficas se utilizó Paint, InfoPath Designer. Estas interfaces se encuentran grabadas en la memoria SD, el formato de imagen debe ser BMP de 24 bits, que es el formato que reconoce el controlador. Una vez encendido el equipo debe ejecutarse un programa 
principal que invoca a diferentes funciones, dependiendo de la opción que escoge el usuario. La variedad de opciones es la siguiente:

- Ingreso de datos vitales.

- Selección del consumo de alimentos.

- Ingreso de datos de glucemia y peso.

- Presentación del historial de glucemia y peso.

- Presentación de resultados.

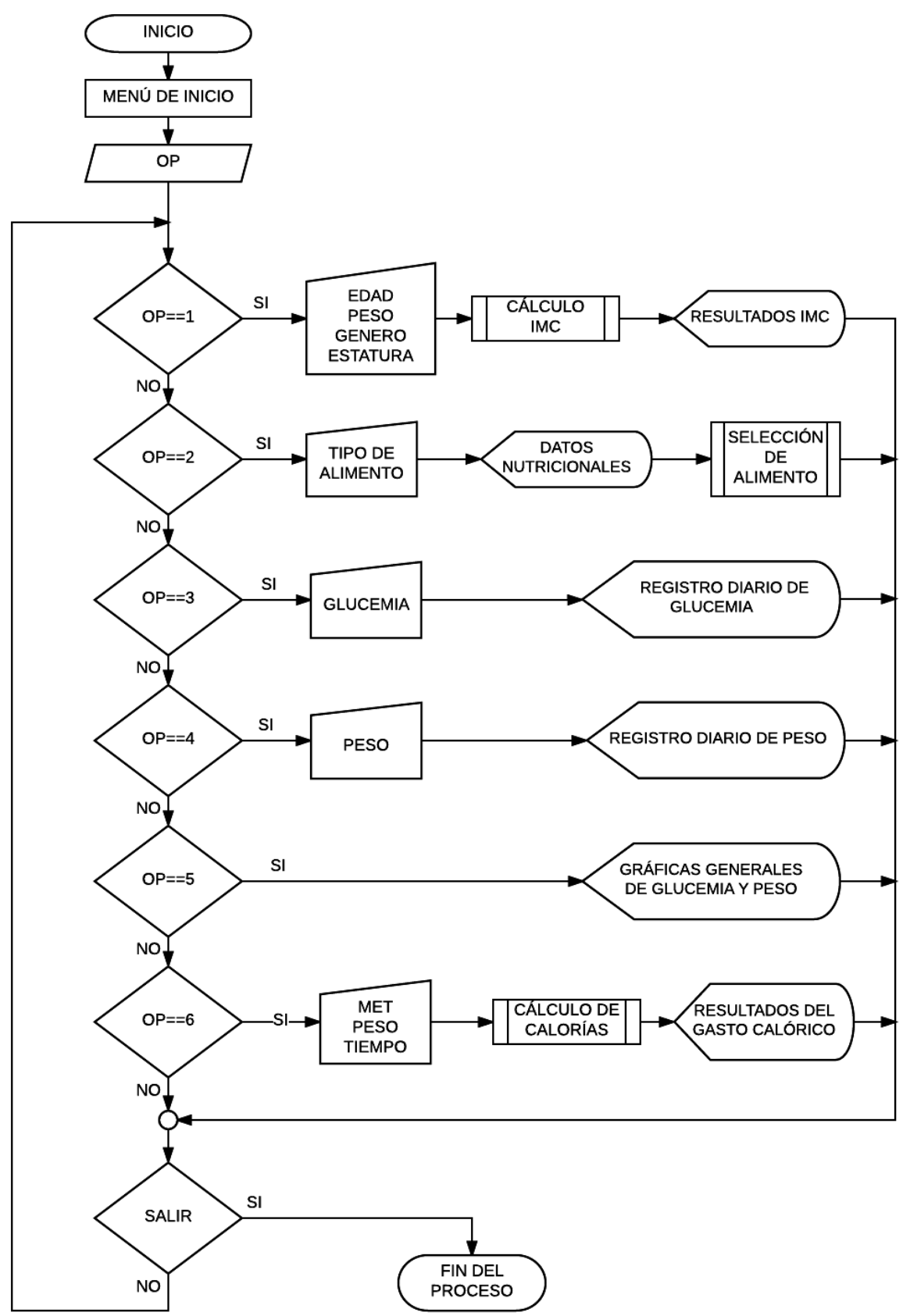

Figura 2. Diagrama de flujo de la operación general del equipo biomédico para control de la nutrición 


\subsubsection{Construcción del prototipo}

Para llegar a construir el prototipo definitivo con microcontrolador AVR XMEGA 256D3 y pantalla táctil de 7 pulgadas, fue necesario escalar los prototipos previos basados en microcontroladores xmega64, xmega192, y pantalla de 4,3 pulgadas. La precisión de la balanza es de 1\%. En la Figura 3 se observa el prototipo.

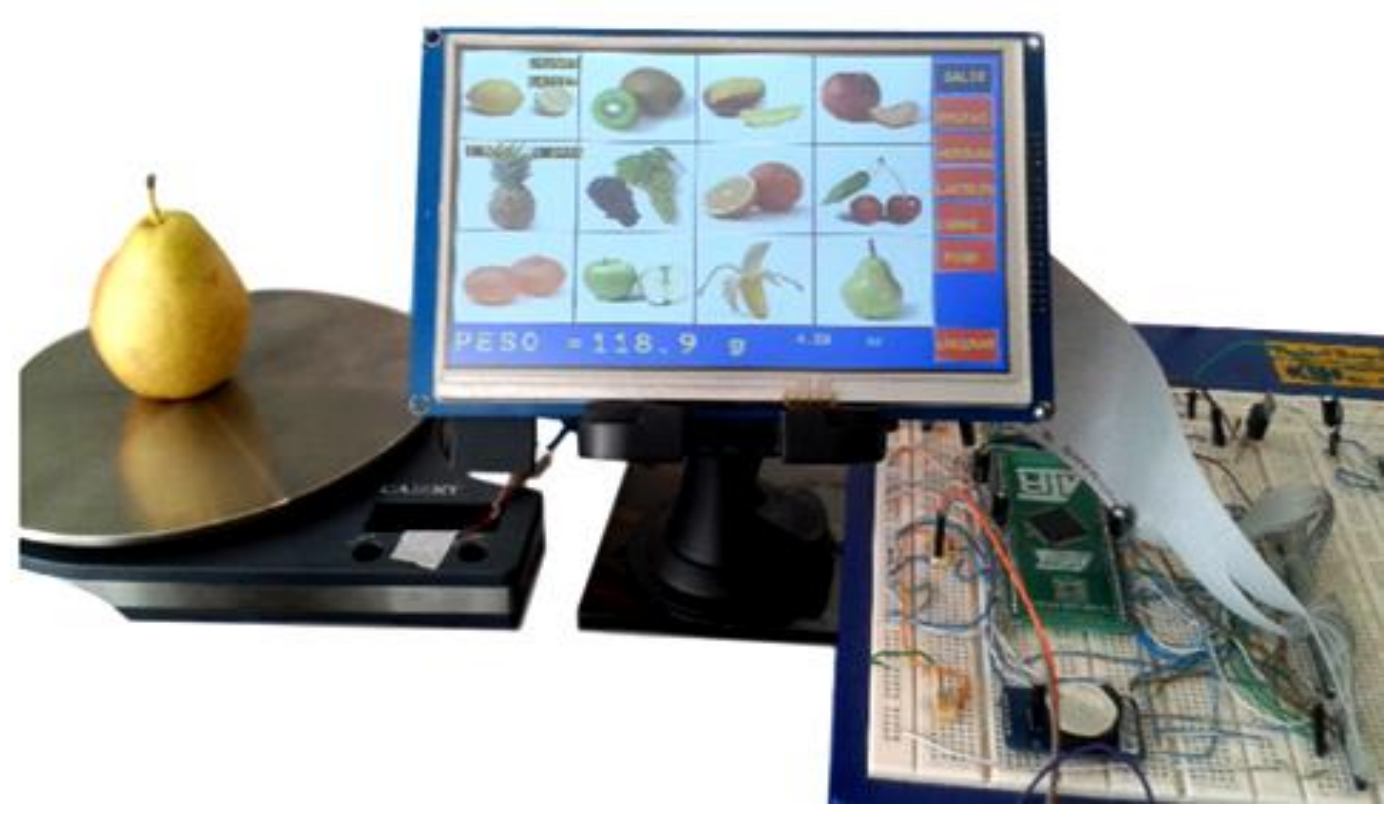

Figura 3. Componentes constitutivos del prototipo de equipo biomédico para el control de la nutrición

El tiempo de barrido de pantalla va de 1 segundo cuando se presenta cada interface, a 2 segundos cuando se toma datos desde la balanza. Se comprobó la capacidad del equipo para recibir, procesar y presentar información de acuerdo al diseño.

\subsection{Desarrollo del equipo para el ejercicio físico de personas con diabetes}

\subsubsection{Materiales y métodos}

\subsubsection{Determinación del grado de afectación del pie diabético}

Los grados de afectación son evaluados y determinados por medio de escalas médicas; una de las más utilizadas a nivel global es la escala de Wagner: En esta escala, las afecciones son clasificadas por grados dependiendo del nivel de severidad. Esta escala puede apreciarse en la Tabla 4. 
Tabla 4. Determinación del grado de afectación según la escala de Wagner

\begin{tabular}{|l|l|}
\hline \multicolumn{2}{|c|}{ Determinación del grado de afectación según la escala de Wagner } \\
\hline Grado 0 & Pie en riesgo presenta celulitis con piel intacta. \\
\hline Grado 1 & Ulcera superficial. \\
\hline Grado 2 & Ulcera profunda. \\
\hline Grado 3 & Absceso profundo y generación de Osteomielitis \\
\hline Grado 4 & Aparición de gangrena en determinadas partes del pie como dedos o falanges \\
\hline Grado 5 & Gangrena en todo o gran parte del pie. \\
\hline
\end{tabular}

\subsubsection{Tratamiento del pie diabético}

Existen diferentes métodos para tratar al pie diabético, dependiendo del grado de severidad en la afectación y del criterio médico. El equipo en desarrollo ayuda al movimiento del pie, para favorecer una mayor circulación sanguínea. Existe la posibilidad de que al paciente le cueste mucho mover el pie ya sea por traumas de dolor derivados de la neuropatía diabética o por complicaciones derivadas de edemas, úlceras e isquemia que produce necrosis en los tejidos.

\subsubsection{Diseño y construcción del equipo}

Para el diseño y construcción se utilizó especificaciones propias de los métodos para el tratamiento del pie diabético, la facilidad de uso, minimización de riesgos de accidente o lesión para el usuario.

Las especificaciones técnicas del equipo son las siguientes:

- Capacidad de movimiento con tres grados de libertad

- Posibilidad de escoger diferentes secuencias de movimiento, las cuales servirán como las terapias coadyuvantes en el tratamiento integral. La ejecución de las secuencias será por medio del comando de máquinas eléctricas y mecanismos.

- Facilidad de uso con interfaz gráfica en pantalla táctil

- Velocidad de respuesta electrónica del sistema microprocesador equivalente al menos a la frecuencia de $20 \mathrm{MHz}$

- Velocidad de respuesta mecánica, equivalente a un tiempo de respuesta en el orden de los milisegundos, con precisión y suavidad en el arranque, marcha y detención del movimiento de los mecanismos

\subsubsection{Definición del prototipo}

Se hace uso de diferentes tarjetas electrónicas microprocesadas, pantallas táctiles y servomotores, para crear el prototipo del sistema hardware - software. Se crea prototipos virtuales y físicos para analizar el cumplimiento de especificaciones mecánicas. Con la integración de 
sistemas, se creará un prototipo mecatrónico a escala reducida para realizar pruebas. Con esto se podrá construir el equipo que utilizará el enfermo de pie diabético.

\subsubsection{Diseño del hardware, del software y del sistema mecánico}

Se aplica la metodología establecida para diseñar cada subsistema del equipo en función de las especificaciones técnicas resultado de los requerimientos.

\subsubsection{Diseño del hardware}

El equipo posee una interfaz gráfica en pantalla táctil color de 5 pulgadas que permite al usuario dar las instrucciones necesarias para que este funcione de acuerdo a su requerimiento médico. La unidad de control implementada en una tarjeta microprocesador envía señales de control a los actuadores para que el mecanismo proporcione los movimientos requeridos. En Figura 4, se puede apreciar la forma en que se integran los componentes y la dirección del flujo de información, señales de control y suministro de energía electica.

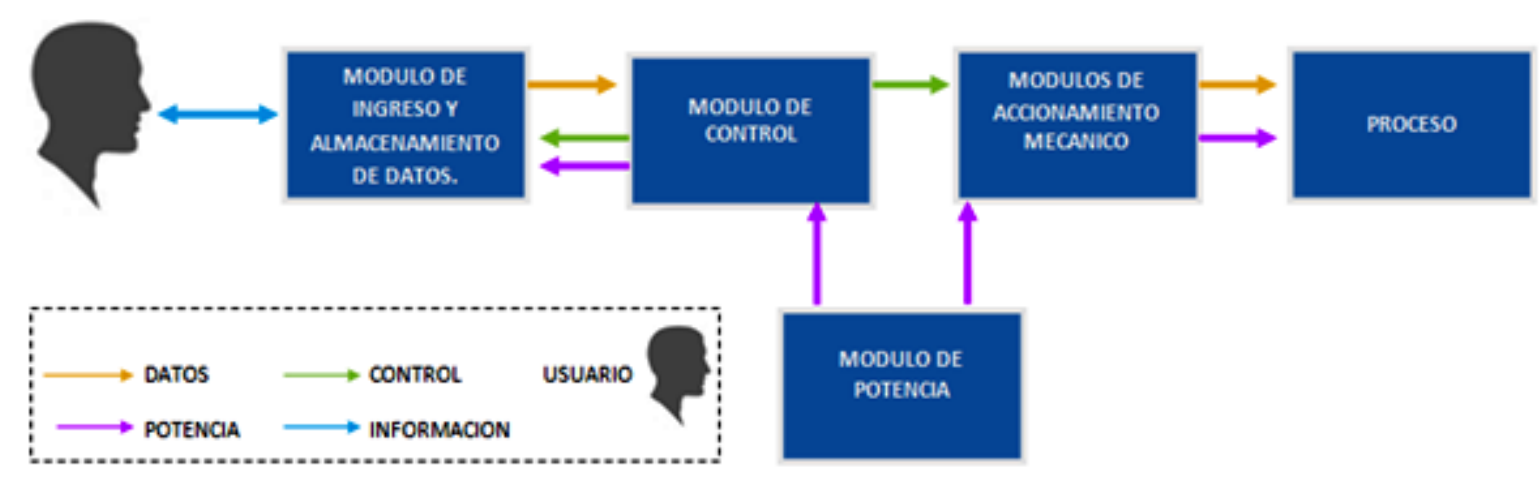

Figura 4. Diagrama de bloques de operación e integración de los componentes - equipo para el ejercicio físico de personas diabéticas

\subsubsection{Diseño del software}

El usuario puede seleccionar alguna de las secuencias establecidas de movimiento por el profesional médico, y establecer el tiempo que realizará la terapia. A continuación, en la Figura 5 se presenta el diagrama de flujo de las operaciones del software. 


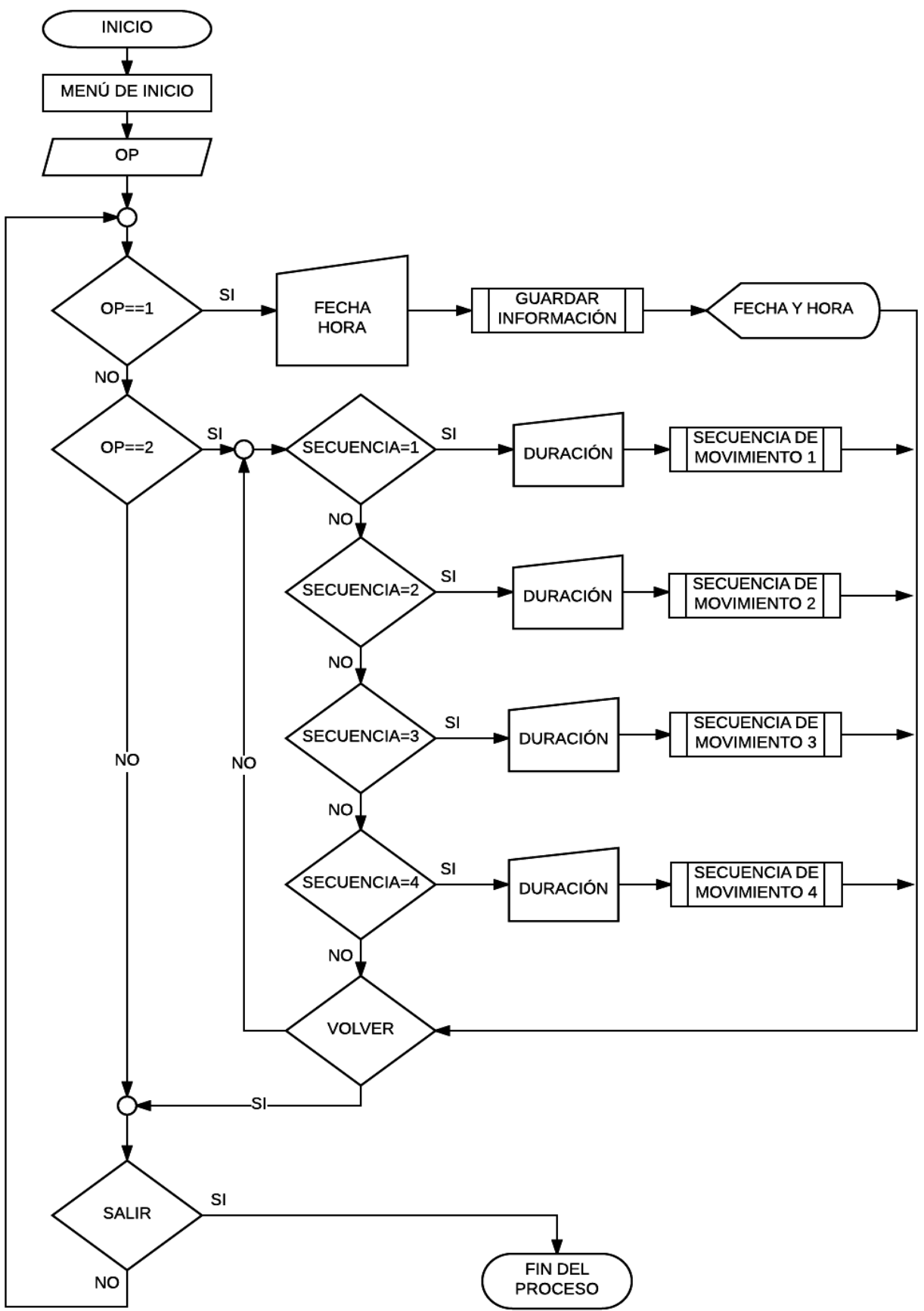

Figura 5. Diagrama de flujo del software - equipo para el ejercicio físico de personas diabéticas

\subsubsection{Diseño virtual del sistema mecánico}

Con el uso de SolidWorks, se analizó diversos prototipos virtuales hasta obtener aquel que satisfacía las especificaciones de diseño previstas. En la Figura 6, se presenta el mecanismo virtual que cumple con los tres movimientos de carácter terapéutico, sujetos a los tres grados de libertad de tipo rotacional. 


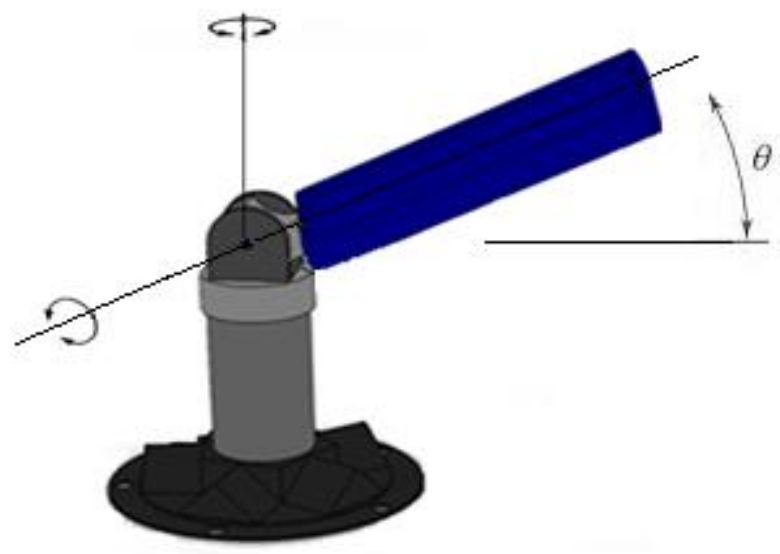

Figura 6. Prototipo virtual del componente mecánico para el equipo ejercitador de extremidades inferiores de personas con diabetes

\section{Resultados y análisis}

\subsection{Equipo para el control de la nutrición de personas con diabetes}

Una vez realizadas satisfactoriamente las pruebas en el prototipo, se procedió a la construcción del equipo. En la Figura 7 puede observarse el equipo biomédico diseñado y construido, una vez que fue terminado. Los puertos de energización y comunicación se encuentran en el costado lateral izquierdo, el ángulo de inclinación de la pantalla considera aspectos ergonómico, la base es amplia y soporta adecuadamente el peso del equipo electrónico desarrollado. Este equipo será utilizado por el enfermo diabético.



Figura 7. Equipo biomédico coadyuvante para control de la nutrición 
El planteamiento y desarrollo del equipo fue validado con la aplicación a un caso de estudio de un paciente diabético. El lapso de tiempo escogido para la presentación de datos glucémicos del paciente, fue de un mes, ya que en este período se pudo determinar la capacidad del mismo, para el manejo y control de su enfermedad.

\subsection{Equipo para el ejercicio físico de personas con diabetes}

El correcto funcionamiento de los sistemas de hardware y software, unido al análisis de la simulación virtual, permitió construir el prototipo físico del sistema mecánico, que al ser integrado a los dos anteriores, dio como resultado el prototipo esperado de equipo biomédico para el tratamiento del pie diabético. En este equipo se realizan pruebas con especificaciones médicas y estándares de calidad que harán posible construir el equipo para la realización de ejercicio físico. En la Figura 8 puede observarse el prototipo físico con sus componentes, organizados en correspondencia con el diagrama de bloques presentado en la Figura 4.

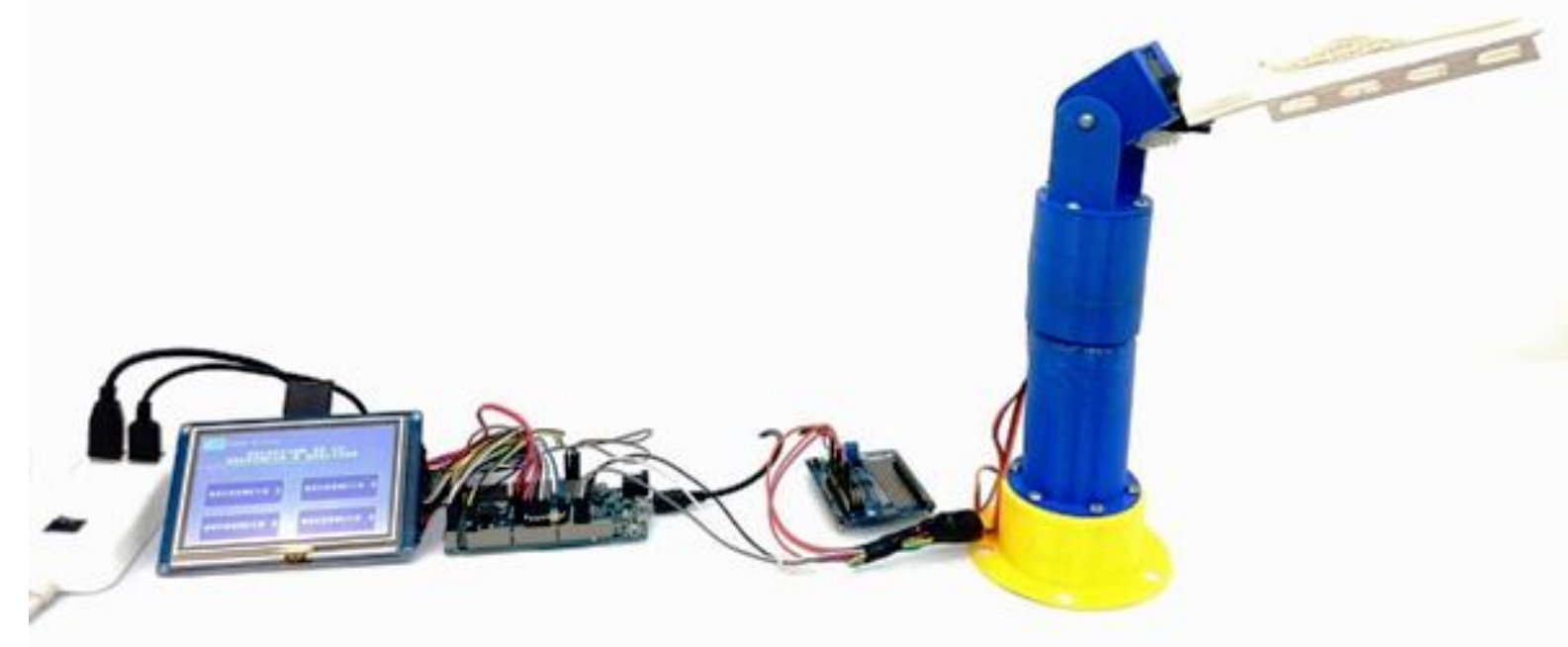

Figura 8. Prototipo físico del equipo biomédico para el ejercicio de personas con diabetes

\section{Conclusiones}

\subsection{Equipo biomédico para el control de la nutrición de personas con diabetes}

- Se cumplió el objetivo de crear un equipo biomédico inédito coadyuvante para el control de la nutrición de personas diabéticas.

- La metodología utilizada fue adecuada para el cumplimiento del objetivo previsto, es decir, el diseño, la construcción del prototipo, y finalmente, la realización del equipo biomédico terminado, para el uso de personas con diabetes. 


\subsection{Equipo biomédico para el ejercicio físico de personas con diabetes}

- La metodología y los estándares de calidad que guiaron este trabajo hicieron posible crear un prototipo inédito, válido para continuar con la investigación, análisis y pruebas para el desarrollo exitoso de un equipo coadyuvante en el tratamiento del pie diabético, con lo cual se cumple el objetivo previsto del desarrollo del prototipo.

- La utilización de hardware, software, materiales y otros dispositivos de alta tecnología, como son el caso de impresoras 3D, permitieron crear un prototipo de alta calidad.

\section{Bibliografía}

FID. (2015). Atlas de la Diabetes de la FID (7ma ed.). Bruselas, Bélgica. Obtenido de http://www.diabetesatlas.org/resources/2015-atlas.html

García Yanes, J. (2009). Tesis Doctoral. Relación entre el índice glucémico, carga glucémica y fibra con la resistencia a la insulina en la población canaria. Tenerife, España.

IDF. (19 de Octubre de 2015). International Diabetes Federation. Obtenido de http://www.idf.org/who-we-are

INEC. (27 de Octubre de 2014). Instituto Nacional de Estadística y Censos. Obtenido de http://www.ecuadorencifras.gob.ec/institucional/home/

Jesús, F. R. (2010). Pie Diabetico Atencion Integral. Mexico,D.F: Mc Graw Hill.

Joslin. (10 de Julio de 2016). Diabetes and nutrition. Obtenido de http://www.joslin.org/info/how_does_fiber_affect_blood_glucose_levels.html

Lineaysalud. (2009). Obtenido de http://www.lineaysalud.com/que-es/metabolismo-basal

Proctor, J. E. (2007). Diabetes Mellitus. Barcelona: Lippincott Williams Wilkins.

Villarino. (09 de Octubre de 2016). Sociedad Española de Dietética y Ciencias de la Alimentación. Obtenido de http://www.nutricion.org/publicaciones/pdf/antropometria/METABOLISMO\%20ENERGETIC O.VILLARINO.pdf

WHO, \& FAO. (2003). Diet, nutrition and the prevention of chronic diseases. WHO Technical report series $916,1-160$. 